\title{
Median and ulnar neuropathy in the guinea-pig
}

\author{
PAMELA M. FULLERTON ${ }^{1}$ AND R. W. GILLIATT \\ From the Institute of Neurology, Queen Square, London
}

In the course of some earlier experiments involving the measurement of conduction velocity in the motor nerves of the guinea-pig, it was discovered by chance that conduction in the median nerve was frequently abnormal in animals which were more than 18 months old. Subsequently it became apparent that this was due to a local lesion of the median or the ulnar nerve at the wrist, which could develop during adult life in otherwise healthy animals (Fullerton and Gilliatt, 1966). This phenomenon has now been further investigated.

\section{METHODS}

Healthy male and female guinea-pigs were used. Most of the young animals, aged 4-8 months, were bred in our own laboratory. Old guinea-pigs were obtained from the Wellcome Veterinary Research Station, from Allington Farm, Porton Down, and from the National Institute for Medical Reseach. These animals were from discarded breeding stock, and their ages ranged from 21 to 30 months.

Animals were fed on a standard SG1 pellet diet (Short and Gammage, 1959) supplemented with fresh cabbage. They were kept in large pens with sawdust flooring to exclude the possibility of trauma to peripheral nerves such as is known to occur when guinea-pigs are kept for long periods on wire-mesh flooring (Fullerton and Gilliatt, 1967).

Maximal motor conduction velocity was measured in anaesthetized animals using a technique similar to that originally developed for the hind limb by Kaeser and Lambert (1962) and described in detail by Fullerton (1966). The median and ulnar nerves were stimulated by stainless steel needles inserted through the skin just above the wrist and in the axilla. Both of these nerves contribute to the motor innervation of the muscles of the forefoot, and since they lie close together at the wrist, it was usually necessary to divide one nerve before stimulating the other, in order to ensure that no cross-stimulation occurred.

In early experiments the conduction distance was first measured on the skin with the stimulating needles in situ; the median and ulnar nerves were then excised and the conduction distance remeasured. It was found that direct measurement on the skin did not give rise to a significant error in the case of the median nerve. In the case of the

'Part-time member of the Scientific Staff, M.R.C. Toxicology Unit, Carshalton, Surrey. ulnar nerve, however, direct measurement between the stimulating electrodes underestimated the true length of the nerve by approximately $5 \mathrm{~mm}$. In later experiments only direct measurement on the skin was used. In these animals the conduction distance for the ulnar nerve was corrected by the addition of $5 \mathrm{~mm}$. before conduction velocity was calculated.

Action potentials from the small muscles of the forefoot were recorded through stainless steel needles with their tips just penetrating the skin. The active recording electrode was placed over the belly of an appropriate muscle and the remote electrode over one of the digits. Since the arrangement of muscles in the forefoot is a complex one (Alezais, 1902), it was necessary in pathological cases to search carefully with the active electrode before accepting that nerve stimulation had failed to activate motor units in any of the intrinsic muscles. In some experiments a double-core recording needle (Disa no. $13 \mathrm{~K} 80$ ) was used to identify single motor units after the belly-tendon records had been obtained.

Intramuscular temperature in the forelimb was recorded at the end of each experiment. It ranged from $35^{\circ} \mathrm{C}$. to $39^{\circ} \mathrm{C}$. in different animals.

Nerves for histological examination were removed using a dissecting microscope and were then slightly stretched on card frames and fixed in Flemming's solution or $10 \%$ formol-saline. Nerves fixed in Flemming's solution were embedded in paraffin; transverse sections were cut at $5 \mu$, and sections stained by the modified Weigert method to demonstrate myelin sheaths (Gutmann and Sanders, 1943). Some sections were counterstained by the van Gieson method. Nerves fixed in formolsaline were examined in two ways. In most cases the nerves were stained with $1 \%$ osmium tetroxide and teased apart in glycerin using the technique described by Thomas (1955); single fibres were prepared under a dissecting microscope at a magnification of $x 100$ and abnormal fibres preserved by mounting in Canada balsam for later examination at higher magnification. In a few cases formol-fixed nerves were embedded in paraffin; longitudinal and transverse sections were stained by the Holmes silver method for axons, and by luxol fast blue for myelin (McDonald, 1963).

\section{ELECTROPHYSIOLOGICAL RESULTS}

In young animals aged 4-8 months maximal motor conduction velocity between axilla and wrist ranged from 37 to $50 \mathrm{~m}$./sec. for the median nerve and 
from 43 to $59 \mathrm{~m}$./sec. for the ulnar nerve. With stimulation just above the wrist the distal latency for the fastest fibres ranged from 0.9 to $1.5 \mathrm{msec}$. for the median nerve and from 1.0 to $1.6 \mathrm{msec}$. for the ulnar nerve. Mean values and standard deviations are given in Table $\mathbf{I}$.

\section{TABLE I}

CONDUCTION VELOCITY AND DISTAL LATENCY IN YOUNG AND OLD GUINEA-PIGS

\begin{tabular}{cccccc} 
Group & $\begin{array}{c}\text { Nerve } \\
\text { Examined }\end{array}$ & $\begin{array}{c}\text { No. of } \\
\text { Cases }\end{array}$ & $\begin{array}{c}\text { Age in } \\
\text { Months }\end{array}$ & $\begin{array}{c}\text { Conduction } \\
\text { Velocity } \\
\text { (m./ sec.) }\end{array}$ & $\begin{array}{c}\text { Distal } \\
\text { Latency } \\
\text { (msec.) }\end{array}$ \\
\hline Young & Median & 14 & $5.5 \pm 1.02$ & $44.3 \pm 3.62$ & $1.2 \pm 0.19$ \\
Old & Median & 13 & $24.8 \pm 3.56$ & $38.3 \pm 8.93$ & $2.4 \pm 0.79$ \\
Young & Ulnar & 14 & $5.9 \pm 1.17$ & $50.5 \pm 4.89$ & $1.3 \pm 0.18$ \\
Old & Ulnar & 12 & $24.1 \pm 3.55$ & $36.7 \pm 4.98$ & $2.8 \pm 1.01$
\end{tabular}

No difficulty was experienced in recording a muscle response to nerve stimulation in any of the young animals examined. In old animals aged 21-30 months, however, median nerve stimulation frequently failed to elicit a muscle response. This occurred in 16 of the 42 median nerves from old animals; in nine of them the ulnar nerve was also inexcitable. When muscle action potentials were evoked by nerve stimulation in old animals, the latency of the response was often increased and its amplitude reduced; sometimes the response was prolonged and polyphasic. Illustrative examples are shown in Figures 1 and 2.

Mean values for maximal motor conduction velocity and distal latency in old animals are shown in Table I. Comparison with the results for young animals shows that the most marked change in the old group was in distal latency, the mean values of $2.4 \mathrm{msec}$. and $2.8 \mathrm{msec}$. for the median and ulnar nerves respectively being at least double the mean values for the same nerves in young animals. Conduction between axilla and wrist was less affected, the reduction in velocity in old animals compared with young ones being less than $30 \%$. Individual results are shown graphically in Fig. 3 in which the limits of the normal range for young animals are drawn as interrupted lines. It can be seen that the most common abnormality in the old animals was an increased distal latency accompanied by a reduced conduction velocity proximal to the wrist. This combination was present in six median and 10 ulnar nerves. However, there were four median nerves and one ulnar nerve in which distal latency was significantly increased although conduction velocity in the proximal part of the nerve was normal. There was no case in which conduction velocity above the wrist was significantly reduced in a nerve with a normal distal latency.

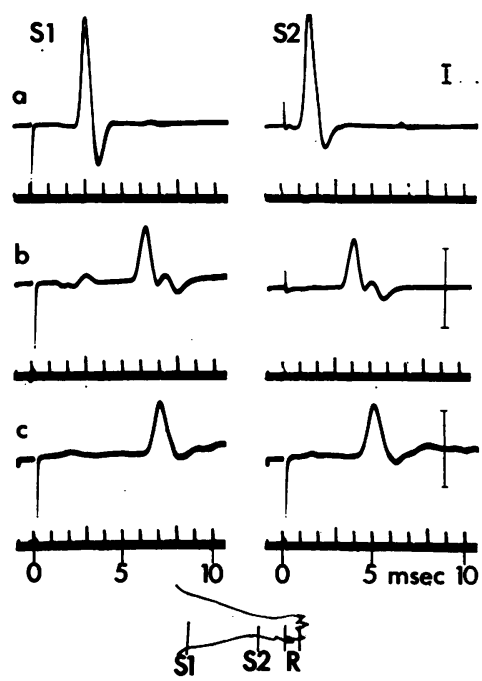

FIG. 1. Muscle action potentials from the forefoot evoked by stimulation of the median or ulnar nerve in the axilla (S1) or at the wrist (S2).

(a) Median nerve stimulation in a 7-month-old animal $\square$ (GP 164).

(b) Median nerve stimulation in a 30-month-old animal (GP 167).

(c) Ulnar nerve stimulation in a 30-month-old animal (GP 166). Cal. $1 \mathrm{mV}$.

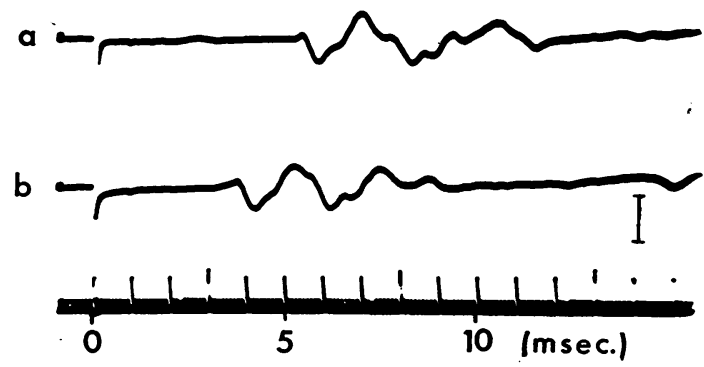

FIG. 2. Delayed and dispersed muscle action potentials recorded from the forefoot after median nerve stimulation, (a) in the axilla, (b) at the wrist, in a 21-month-old animal (GP 182). Cal. $500 \mu \mathrm{V}$. 


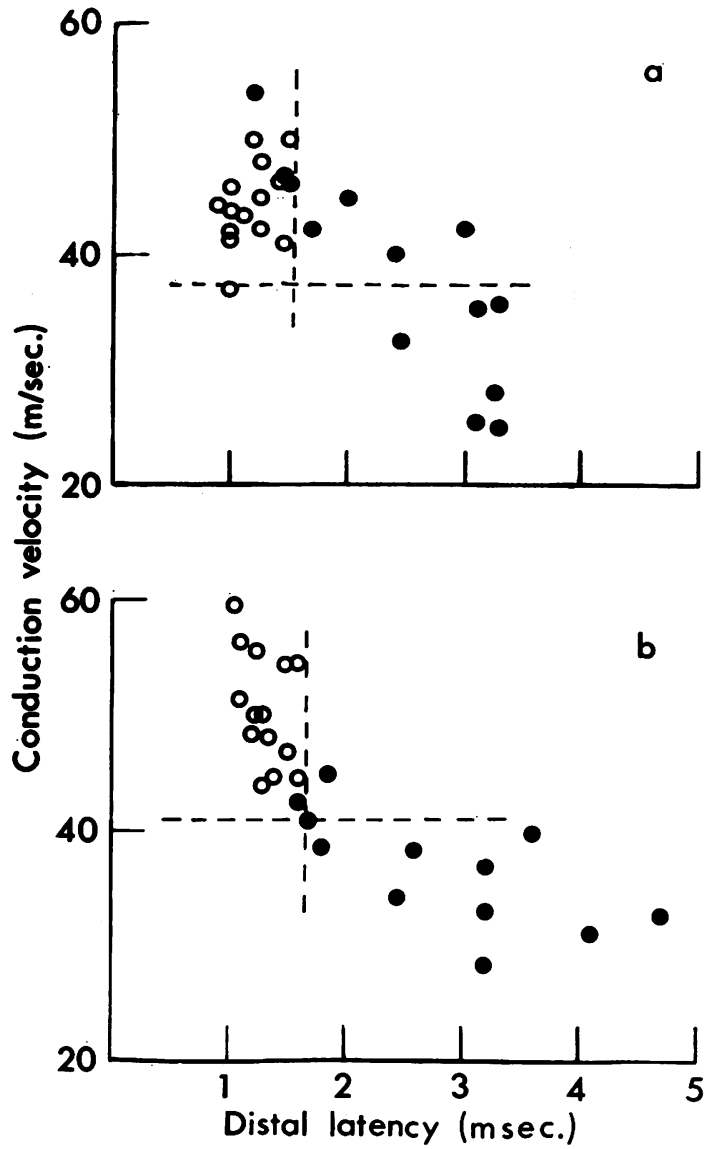

FIG. 3. Conduction velocity proximal to the wrist plotted against distal latency in young (open circles) and old guinea-pigs (filled circles). The vertical interrupted line is $2 S . D$. above the mean for distal latency in young animals, and the horizontal interrupted line is 2 S.D. below the mean for proximal velocity. Results for the median nerve are shown in a and for the ulnar nerve in $\mathrm{b}$.

These results suggested the possibility of a local lesion in the distal part of the nerve, and confirmation of this was obtained from the study of animals in which the muscle response to nerve stimulation was prolonged and polyphasic. In such cases the use of a small double-core needle electrode showed that the late components of the polyphasic response were due to single motor units with greatly increased distal latencies. These longlatency units were identified by their all-or-none response to changes in stimulus intensity, and by their constant waveform with nerve stimulation at two levels. When their latencies were compared with those of the earliest units to respond, it was clear that the extra delay was occurring distal to the wrist

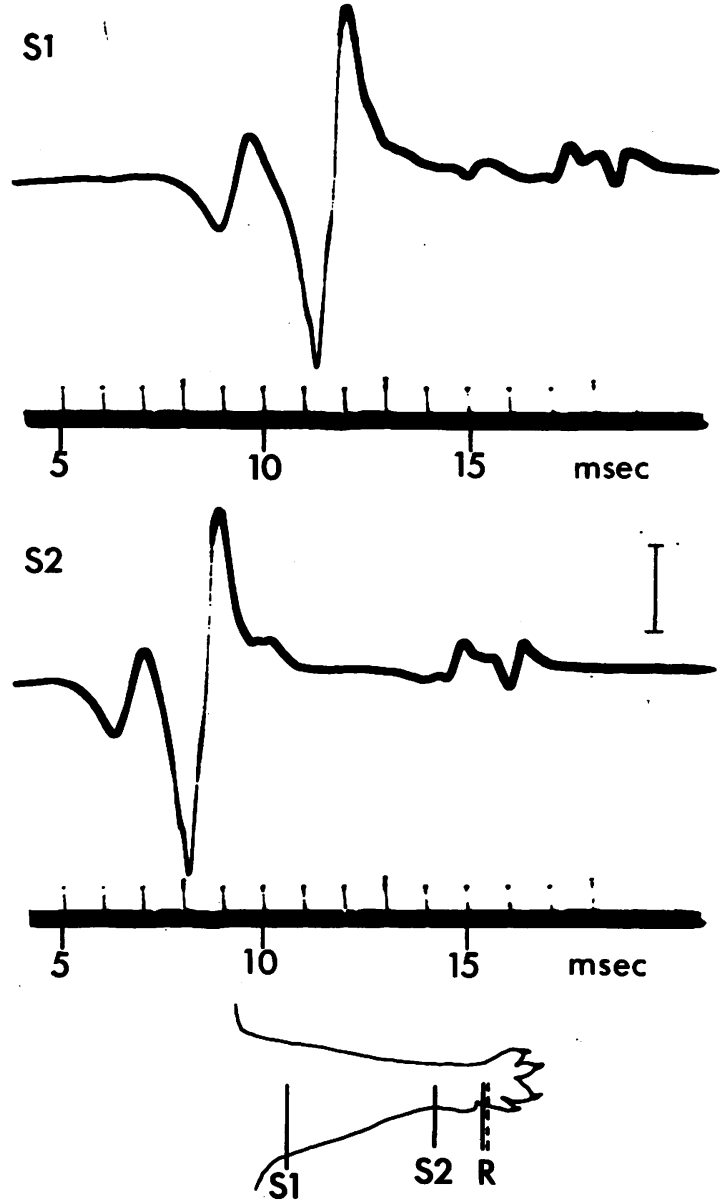

FIG. 4. Early and late potentials from the forefoot in response to stimulation of the median nerve in the axilla (SI) and at the wrist (S2) in a 21-month-old animal (GP 177). The time scale indicates the interval after the stimulus in each case. A double-core needle electrode was used for recording. Cal. $500 \mu \mathrm{V}$.

electrode. A typical record is shown in Fig. 4 and results from this and from other old animals are shown diagrammatically in Figure 5 . In the examples shown, the distal latencies for the first units to respond ranged from 2.9 to $4.9 \mathrm{msec}$; the late units had distal latencies ranging from 6.4 to 14.4 msec. and yet conduction in their proximal parts was only slightly slowed compared with the early units.

These findings indicate a local abnormality of the median and ulnar nerves at the level of the wrist or in the forefoot itself. The reduced motor conduction velocity found in the proximal parts of the nerves would not be incompatible with a distal lesion, as a similar reduction has been found proximal to other peripheral nerve lesions both in 


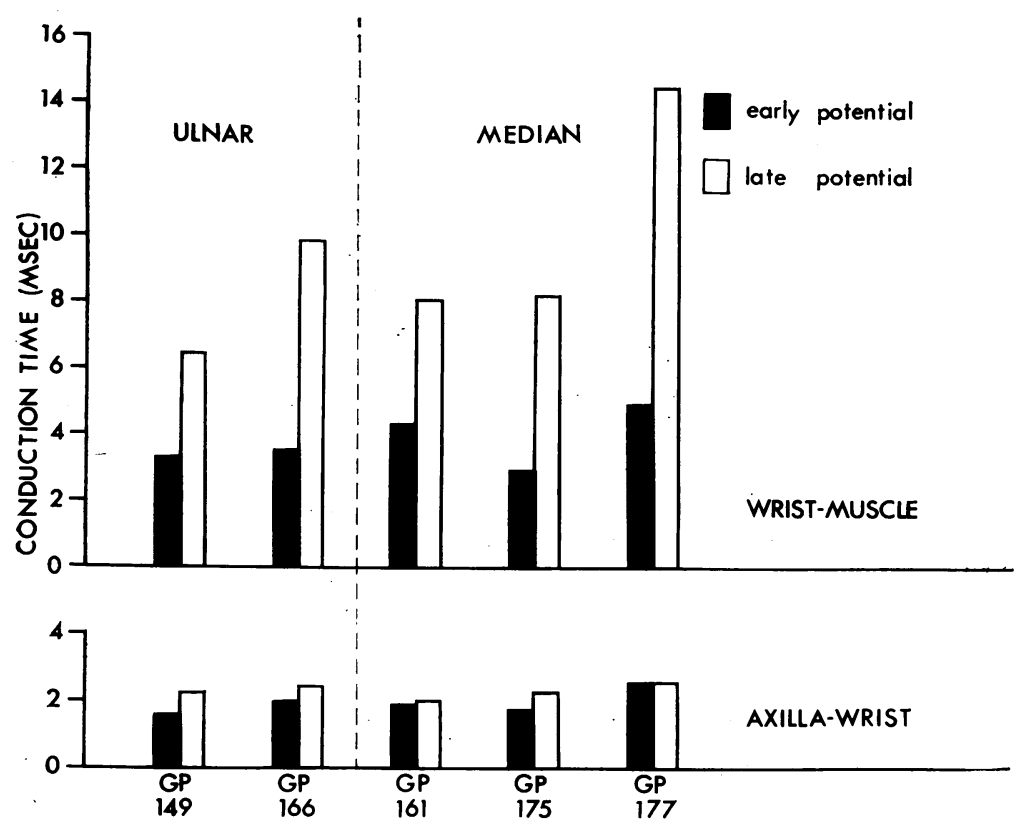

FIG. 5. Conduction times in proximal and distal segments of the median and ulnar nerves for early and late potentials recorded from old animals as shown in Figure 4. man (Ebeling, Gilliatt, and Thomas, 1960; Thomas, 1960) and in animals (Cragg and Thomas, 1961; Fullerton and Gilliatt, 1967).

\section{ANATOMICAL RESULTS}

Dissection of the palmar surface of the wrist in both young and old guinea-pigs revealed a transverse arch made up of fibrous tissue and a cartilaginous bar supporting the foot pad. From Fig. 6 it can be seen that the median nerve passes under the transverse cartilaginous bar with its accompanying artery. Although it is not shown in Fig. 6, the deep branch of the ulnar nerve also passes under the bar on its ulnar side. In old animals the cartilage of the transverse bar was often calcified, and the median and ulnar nerves were involved in dense fibrous tissue. In some of the animals it could be seen that the nerves were thinned at this level. A single fascicle of the median nerve from the region of the wrist in a 26-month-old animal is shown in Figure 7. The specimen shows both thinning and partial loss of myelin staining over a distance of approximately $3 \mathrm{~mm}$.

Transverse sections of the median and ulnar nerves from old animals showed changes which were mainly at the level of the wrist in mild cases, but which extended distally into the forefoot in severe cases. As an example of the former, sections from a 21-month-old animal are shown in Figure 8. The electrophysiological records which had been ob- tained on stimulating the median nerve in this animal are shown in Fig. 2, and it can be seen that a small delayed and polyphasic response was recorded from the forefoot with stimulation at the wrist. Transverse sections through the nerve at $1 \mathrm{~mm}$. intervals showed almost complete loss of myelin staining at the level of the wrist, myelinated fibres reappearing distally. In the sections shown in Fig. 8 the same fascicles can be seen above the demyelinated region (Fig. 8a), in the upper part of the demyelinated region (Fig. 8b), and below this (Fig. 8c).

A median nerve which was electrically inexcitable is shown in Fig. 9 and it can be seen that the histological changes were much more severe in this case. The fascicles are shrunken and stain deeply with Van Gieson at the level of the lesion (Fig. 9c). No myelinated fibres can be seen at this level or below it (Fig. 9d), although some myelin debris is present in the lower section. Above the thinned segment there is endoneurial swelling and thickening of the perineurial sheaths (Fig. 9b). At a higher level the fascicles are probably normal in size (Fig. 9a) but the number of myelinated fibres is considerably reduced. At this level there were occasional fascicles containing a normal number of fibres but serial sections showed that these left the main nerve trunk before it passed under the transverse arch at the wrist. One such fascicle is shown in Fig. 10 together with an affected fascicle from the same section (taken $10 \mathrm{~mm}$. above the wrist). It can 


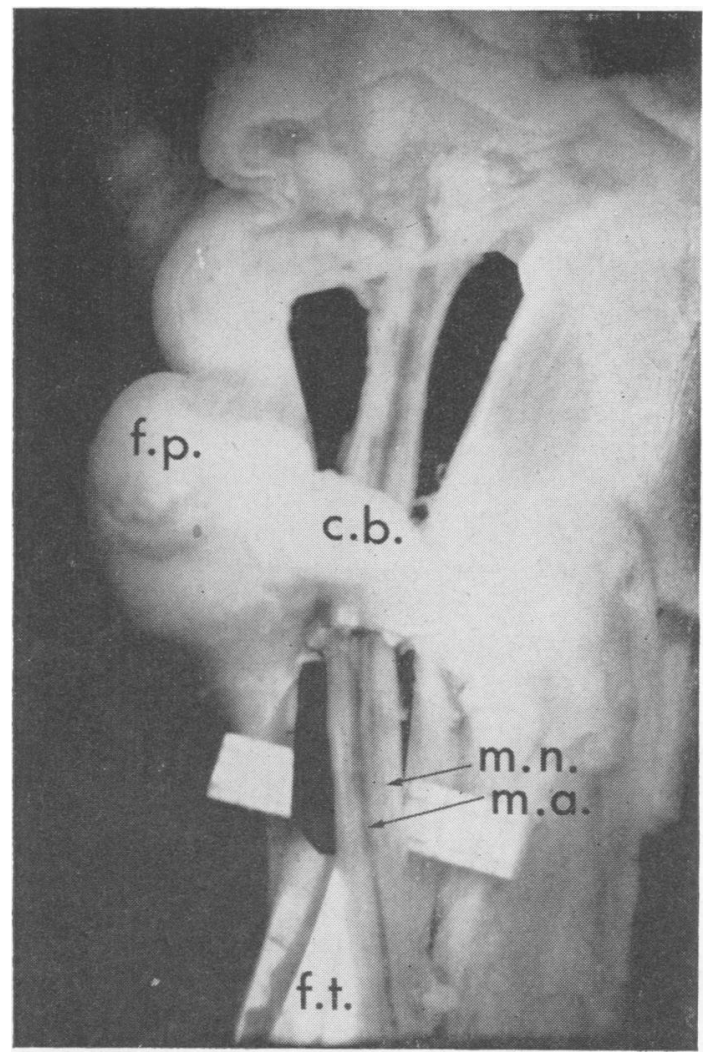

FIG. 6. A dissection of the palmar surface of the right wrist and forefoot of a young guinea-pig. A piece of black card has been placed behind the median nerve (m.n.) and its accompanying artery (m.a.). The transverse cartilaginous bar (c.b.) which supports the foot pad (f.p.) can be seen. Proximal to the wrist a card marked with $1 \mathrm{~mm}$. squares has been inserted in front of the flexor tendons (f.t.). (By courtesy of Dr. J. E. C. Hern).

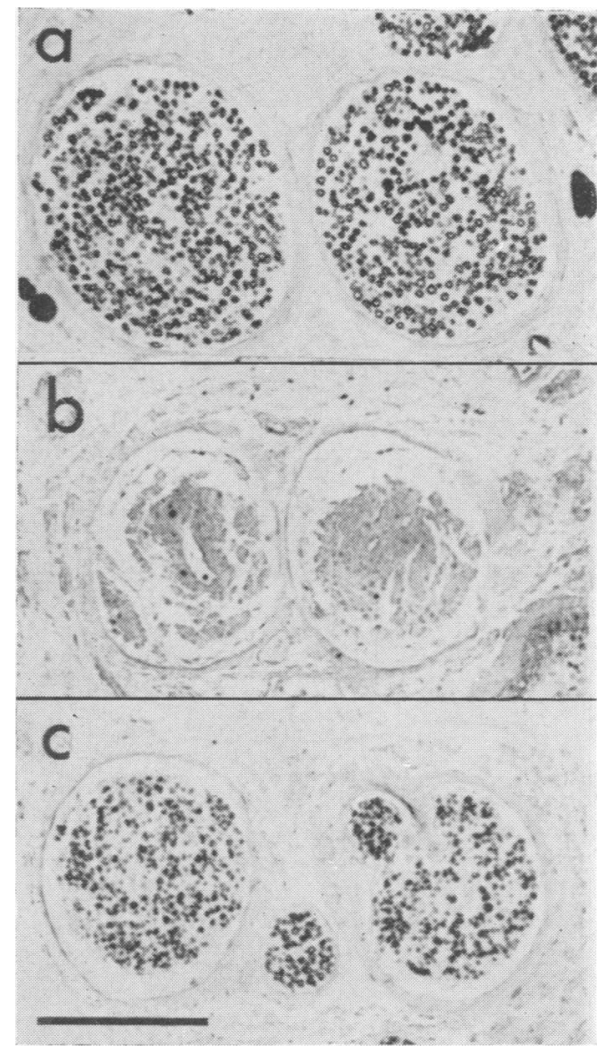

FIG. 8. GP 182. Two fascicles of the median nerve from transverse sections taken (a) above, (b) at, and (c) below the wrist. The distance between a and $\mathrm{b}$ was $2 \mathrm{~mm}$. and between $\mathrm{b}$ and c $3 \mathrm{~mm}$. Sections fixed in Flemming's solution and stained by modified Weigert method to show myelin sheaths. Scale-200 $\mu$.

FIG. 7. GP 150, aged 26 months. A single fascicle of the median nerve at the wrist stained with $1 \%$ osmium tetroxide Scale-3 $\mathrm{mm}$. 


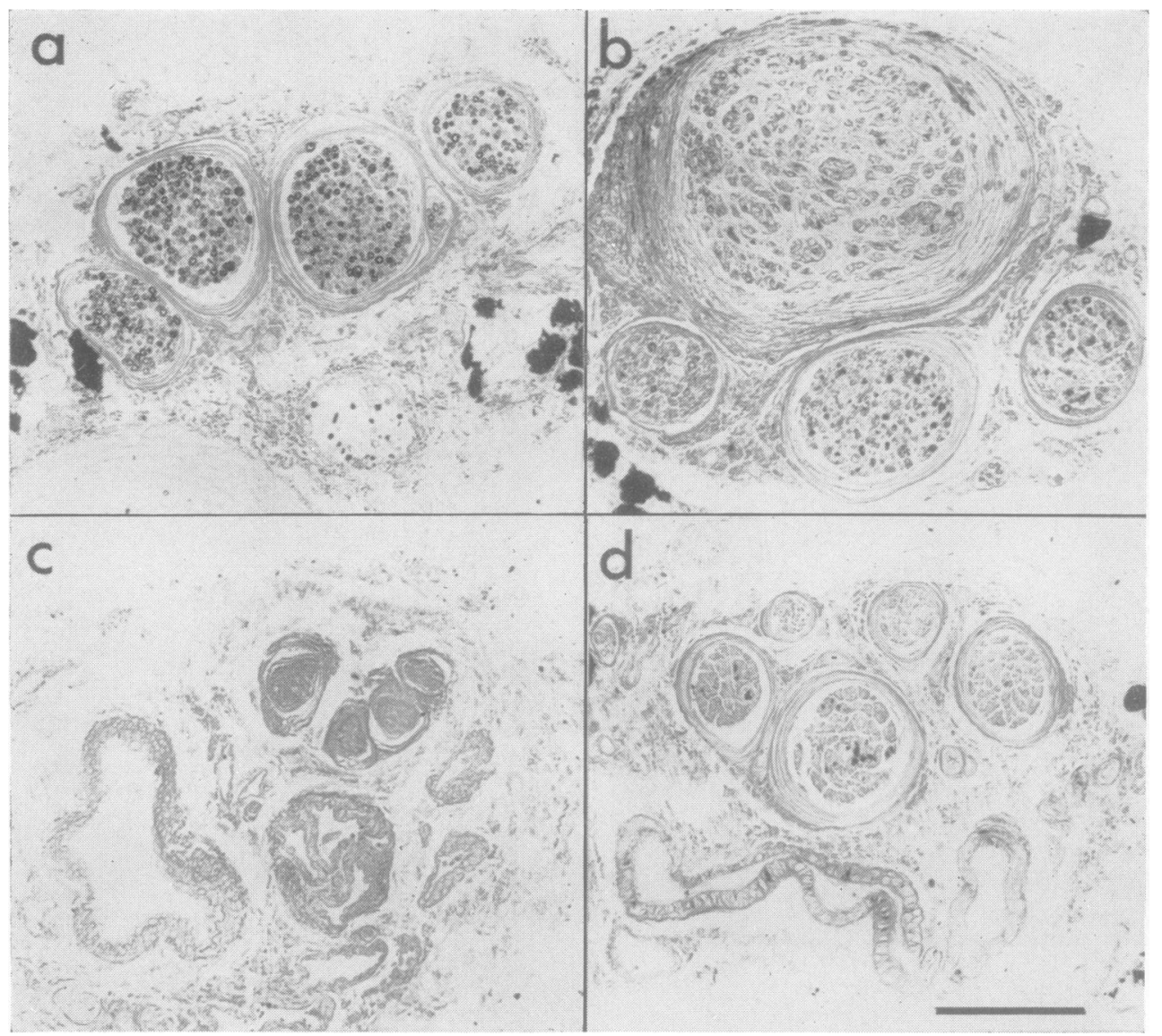

FIG. 9. GP 155, aged 27 months. Four fascicles of the median nerve from transverse sections taken (a) $8 \mathrm{~mm}$. and (b) $4 \mathrm{~mm}$. above the wrist, (c) at the wrist and (d) $8 \mathrm{~mm}$. below it. Fixation and staining as in Fig. 8 with Van Gieson counterstain. Scale-200 $\mu$.

be seen that the nerve fibres from the main nerve trunk are of smaller diameter than those which escaped the lesion by passing superficially.

Partial or complete loss of myelinated fibres from the median nerve at the wrist was found in six old animals. All had shown delayed and dispersed action potentials or complete loss of the muscle response to nerve stimulation. In three of them there was severe loss of myelin at the wrist with some recovery below. In the remainder transverse sections showed no myelinated fibres at the wrist or distal to it.

Electrically inexcitable median nerves from three other animals were used to confirm the presence of axonal loss. Longitudinal and transverse sections stained by the Holmes silver method and counterstained with luxol fast blue showed that a number of fine non-myelinated axons were present distal to the wrist, but few large axons were seen and in some fascicles they were totally absent.

Dissection of the deep branch of the ulnar nerve proved to be extremely difficult in affected animals and for this reason relatively few nerves were examined histologically. In only one case was an electrically inexcitable ulnar nerve successfully obtained with preservation of both deep and superficial branches above and at the level of the wrist. Transverse sections of the deep branch of this nerve showed the complete absence of myelinated fibres at the level of the wrist whereas the superficial branch was normal. There was also a marked difference between the two branches in the forearm, the fibre density being reduced in the deep branch compared with the superficial one.

Transverse sections of the median nerve at the level of the wrist were examined in five young 


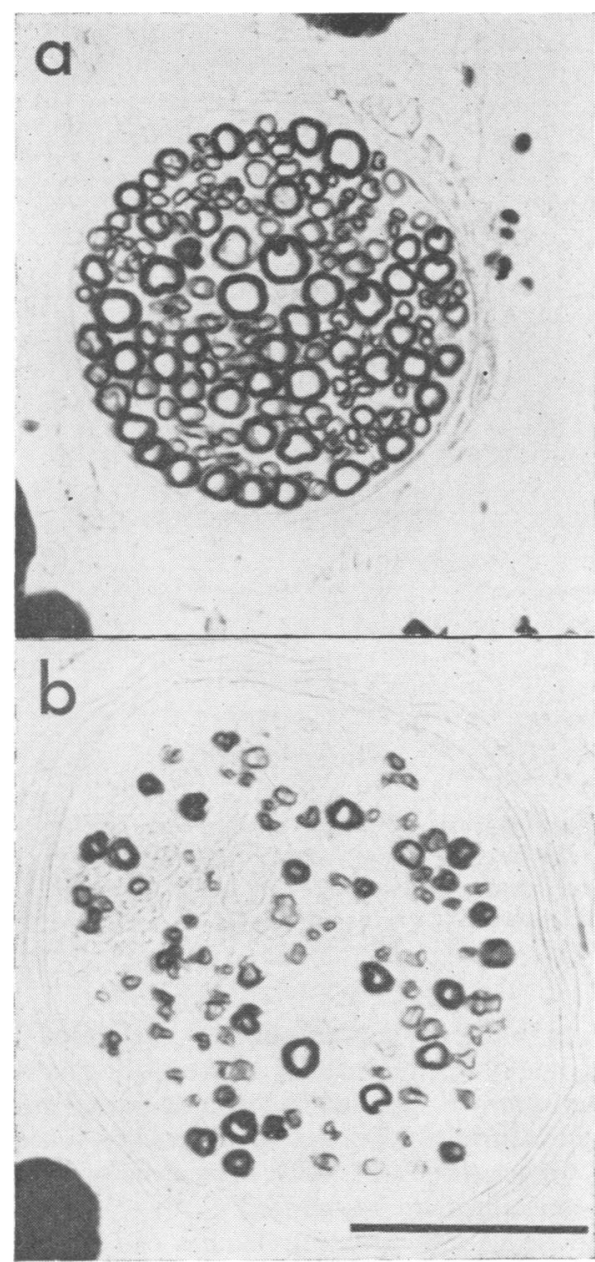

FIG. 10. GP 155. Two fascicles from the same transverse section of the median nerve $10 \mathrm{~mm}$. above the wrist. The fascicle shown in a left the median nerve just below the level of the section. The fascicle shown in $\mathrm{b}$ passed under the cartilaginous bar with the main nerve trunk. Staining as in Figure 8. Scale-50 $\mu$.

guinea-pigs. The number of myelinated fibres was reduced in one fascicle of the median nerve from a 7-month-old animal. In the remainder myelinated fibre density appeared to be normal.

The nature of the lesion in old animals was investigated further by examining single fibres from the median nerve at the wrist. Two of the five nerves examined had been inexcitable on electrical stimulation, and in both cases complete degeneration of myelinated fibres had occurred. Only myelin debris was found at the wrist level and no surviving fibres were seen at this level or distal to it. A number of fibres showed demyelination of parts of the internodal segments above the level of complete degeneration; in some cases this change extended for several segments above it.

The three other old animals from which single fibres were examined had shown slowing of conduction and dispersion of muscle action potentials on nerve stimulation; in these cases single fibres from the wrist showed mainly segmental demyelination. This appeared to affect many fibres at approximately the same level, normal myelin reappearing after a gap of 2 to $3 \mathrm{~mm}$. In the gap there was sometimes complete loss of myelin but a more common finding was early remyelination with the formation of short, thinly myelinated internodes. Demyelination of parts of internodes above or below the main gap was also common. An example can be seen in Fig. 11 which shows consecutive lengths of a single fibre. Between the large arrows a length of nerve $3 \mathrm{~mm}$. long has undergone demyelination and is thinly remyelinated. Distal to this two shorter lengths of abnormal myelin are present and are shown by small arrows.

\section{DISCUSSION}

The lesion which we have described has been recognized electrically and histologically but it is interesting that the animals themselves have shown no abnormality in the use of the forefoot which we have been able to detect. On the sensory side, reaction to pin prick in the foot and toes has been preserved, and no trophic lesions have been seen. These latter findings may be explained by the persistence of fine non-myelinated axons below the level of the lesion. On the present evidence it is not possible to decide whether these are non-myelinated fibres which are unaffected by the lesion, or whether they indicate attempted regeneration. In this context it may be noted that Lehmann and Pretschner (1966) found that $\mathrm{C}$ fibres were often spared in their experimental nerve lesions produced by inserting catgut ligatures through peripheral nerves.

The age of our animals in relation to the life span of the guinea-pig is of some interest. Although we have referred to our 2-year-old group as 'old' animals, it should be remembered that the life span of a guinea-pig under favourable conditions may be much longer than this. Paterson (1967) refers to an active breeding life of $\mathbf{3 0}$ months; Rogers regards guinea-pigs less than 1 year old as young, those aged 1 to 2 years as mature, those aged 2 to 3 years as old, and animals more than $\mathbf{3}$ years old as senile (Covell and Rogers, 1957; Rogers, personal communication). It therefore appears that the peripheral nerve lesions described in the present paper arose in active rather than senile animals. 


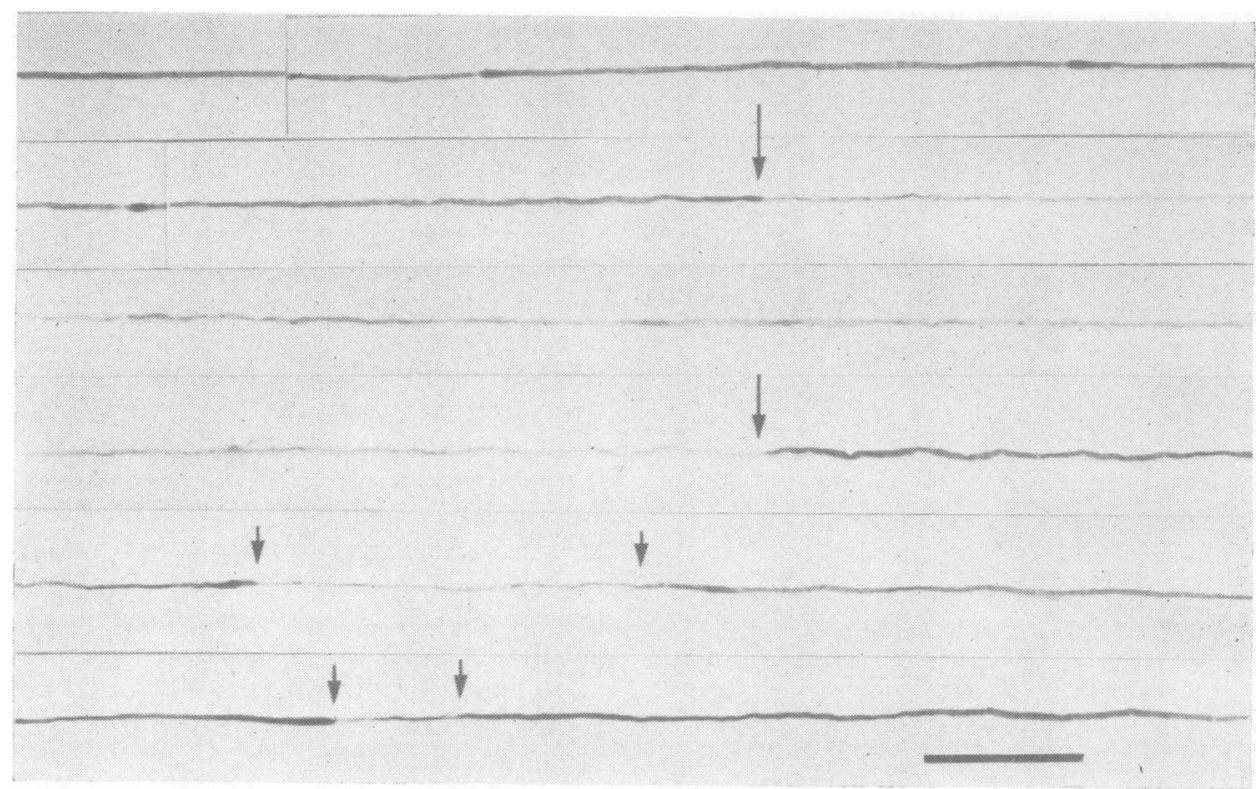

FIG. 11. GP 190, aged 29 months. Consecutive lengths of a single fibre from the median nerve at the wrist. The proximal end of the fibre is at the top. Between the large arrows a length of approximately $3 \mathrm{~mm}$. has undergone demyelination followed by partial remyelination. In the distal part of the fibre two shorter lengths undergoing remyelination are shown by small arrows. $1 \%$ osmium tetroxide. Scale-200 $\mu$.

Since our old animals were from discarded breeding stock, there was initially a preponderance of females but, in order to ensure that the lesion also occurred in males, we subsequently obtained male animals of comparable age and found the incidence of nerve lesions to be similar in the two sexes. In this respect the lesion differs from the carpal tunnel syndrome in man which occurs more commonly in females than in males.

There are other reasons why it would be unwise to press the analogy between this lesion and the human carpal tunnel syndrome too far. We are not merely dealing with a transverse carpal ligament, as in man, but with a cartilaginous bar which becomes calcified in the older animals. The fact that the ulnar nerve passes under the transverse bar is another point of difference from man, and until the effect of surgical removal of the transverse bar has been attempted, it would be unwise to assume that this structure produces nerve constriction in the same way that the transverse carpal ligament does in man.

Whatever the exact mechanism, it is clear that we are dealing with a naturally occurring peripheral nerve lesion which offers a valuable opportunity to study pathological phenomena which also occur in man. At the level of the lesion itself there is thinning of nerve fascicles with either segmental demyelin- ation or Wallerian degeneration of myelinated fibres. Immediately above there is neuroma formation, if this term may be applied to the endoneurial swelling and perineurial thickening which has been observed. At a higher level still, both fibre density and fibre diameter appear to be reduced.

The electrophysiological changes can be satisfactorily explained on the basis of the anatomical findings described above. Segmental demyelination is known to be accompanied by reduced conduction velocity in both generalized and localized neuropathies (Kaeser and Lambert, 1962; McDonald, 1963; Cragg and Thomas, 1964; Mayer and DennyBrown, 1964; Lehmann and Pretschner, 1966; Fullerton and Gilliatt, 1967). Such a reduction in velocity would explain the delayed and dispersed muscle action potentials which were recorded in the present study. In more severely affected animals our failure to activate motor units in the forefoot by electrical stimulation of the median and ulnar nerves is explained by the subsequent finding of complete degeneration of myelinated fibres at the level of the wrist.

Changes in conduction velocity proximal to nerve section or crush have been described previously by several writers (Gutmann and Holubár, 1951 ; Kiraly and Krnjević, 1959; Cragg and Thomas, 
1961). For example, Cragg and Thomas showed in the rabbit that velocity above the suture line fell by about $20 \%$ and returned to normal when regeneration occurred. When regeneration was prevented, velocity in the proximal part of the divided nerve could fall by as much as $40 \%$ within a year. Similar changes in velocity have been reported in the proximal parts of sutured nerves in man (Gilliatt, 1961) and in nerves proximal to chronic peripheral lesions both in man (Ebeling et al., 1960; Thomas, 1960) and in animals (Fullerton and Gilliatt, 1967).

In the case of nerve section and crush, it is known that there is a reduction in the diameter of the largest fibres of the proximal stump (for references see Aitken and Thomas, 1962), but there are few relevant observations on chronic pressure lesions. In regenerating rat nerve subjected to constriction, Weiss and Hiscoe (1948) described axonal swelling immediately above the constricted zone but this did not extend to the more proximal parts of the fibres. Duncan (1948) found a normal fibre diameter above local demyelination produced by ligatures in young rats and kittens. In the single patient with the carpal tunnel syndrome studied by Thomas and Fullerton (1963) the fibre diameter spectrum in the mid-forearm was normal although a pronounced change was present at the wrist itself.

In our guinea-pigs, transverse sections taken 10 $\mathrm{mm}$. above the level of the lesion showed that maximal fibre diameter was markedly reduced when compared with that of adjacent fascicles which had escaped distal compression. Since the fibre density was also reduced, the change in maximal diameter could have been due to the selective loss of large fibres. However, it seems more likely that it was due to a retrograde change in fibres which were compressed distally; this would be in keeping with the previous results of nerve section and crush (Aitken and Thomas, 1962). If the retrograde change extended proximally for a considerable distance, it would explain the reduced conduction velocity which we found in the proximal parts of affected nerves. Further studies are clearly needed to establish this point with certainty

\section{SUMMARY AND CONCLUSIONS}

During adult life, laboratory guinea-pigs may develop a peripheral nerve lesion affecting the median and ulnar nerves at the wrist. The lesion occurs under the transverse cartilaginous bar which supports the footpad, and localized demyelination results. In severely affected animals complete degeneration of myelinated fibres may occur.

Endoneurial and perineurial changes may result in a traumatic neuroma immediately above the lesion. At a higher level in th.e forelimb, the affected nerves show a reduction in both fibre density and fibre diameter, these retrograde changes being most marked in nerves showing the most severe changes at the wrist itself.

The physiological effect of these anatomical changes is to cause either slowing of nerve conduction or a complete conduction block. Slowing of conduction affects mainly the distal parts of the nerve fibres, velocity proximal to the wrist being reduced to a lesser extent.

We are most grateful to Mr. G. J. R. Hovell, Mr. J. S. Paterson and Mr. D. J. Short for providing the old animals used in this study. We are also indebted to Dr. J. E. C. Hern for permission to use Fig. 6, and to Dr. W. I. McDonald for his help and advice. We wish to thank Miss Christine Botwright, Mr. W. F. Hinkes, and Mr. K. Yogendran for technical assistance. A generous grant to one of us from the National Fund for Research into Crippling Diseases is gratefully acknowledged.

\section{REFERENCES}

Aitken, J. T.. and Thomas, P. K. (1962). Retrograde changes in fibre size following nerve section. J. Anat. (Lond.), 96, 121-129.

Alezais, (1902). Etude anatomique du Cobaye. J. Anat. (Paris), 38, 259-275.

Covell, W. P., and Rogers, J. B. (1957). Pathologic changes in the inner ears of senile guinea-pigs. Laryngoscope, 67, 118-129.

Cragg, B. G., and Thomas, P. K. (1961). Changes in conduction velocity and fibre size proximal to peripheral nerve lesions. J. Physiol. (Lond.), 157, 315-327.

- - (1964). Changes in nerve conduction in experimental allergic neuritis. J. Neurol. Neurosurg. Psychiat., 27, 106-115.

Duncan, D. (1948). Alterations in the structure of nerves caused by restricting their growth with ligatures. J. Neuropath. exp. Neurol., 7, 261-273.

Ebeling, P., Gilliatt, R. W., and Thomas, P. K. (1960). A clinical and electrical study of ulnar nerve lesions in the hand. J. Neurol. Neurosurg. Psychiat., 23, 1-9.

Fullerton, P. M. (1966). Chronic peripheral neuropathy produced by lead poisoning in guinea-pigs. J. Neuropath. exp. Neurol., 25, 214-236.

_- and Gilliatt, R. W. (1966). Spontaneous peripheral nerve damage in the guinea-pig. J. Physiol. (Lond.), 183, 52-53P.

- - (1967). Pressure neuropathy in the hind foot of the guinea-pig. J. Neurol. Neurosurg. Psychiat., 30, 18-25.

Gilliatt, R. W. (1961). Nerve conduction: Motor and sensory. In Electrodiagnosis and Electromyography, 2nd ed., edited by S. Licht, 385-411. Licht, New Haven, Connecticut.

Gutmann, E., and Holubár, J. (1951). Atrophy of nerve fibres in the central stump following nerve section and the possibilities of its prevention. Arch. int. Studi neurol., 1, 314-324.

- and Sanders, F. K. (1943). Recovery of fibre numbers and diameters in the regeneration of peripheral nerves. J. Physiol. (Lond.), 101, 489-518.

Kaeser, H. E., and Lambert, E. H. (1962). Nerve function studies in experimental polyneuritis. Electroenceph. clin. Neurophysiol., suppl. 22, 29-35.

Kiraly, J. K., and Krnjević, K. (1959). Some retrograde changes in function of nerves after peripheral section. Quart. J. exp. Physiol., 44, 244-257.

Lehmann, H. J., and Pretschner, D. P. (1966). Experimentelle Untersuchungen zum Engpasssyndrom peripherer Nerven. Disch. $Z$. Nervenheilk., 188, 308-330.

McDonald, W. I. (1963). The effects of experimental demyelination on conduction in peripheral nerve: A histological and electrophysiological study. Brain, 86, 481-524.

Mayer, R. F., and Denny-Brown, D. (1964). Conduction velocity in peripheral nerve during experimental demyelination in the cat. Neurology (Minneap.), 14, 714-726. 
Paterson, J. S. (1967). The guinea-pig or cavy. In The UFAW Handbook on the Care and Management of Laboratory Animals, edited by the staff of UFAW (Universities Federation for Animal Welfare) 3rd ed., 241-287. Livingstone, Edinburgh and London.

Short, D. J., and Gammage, L. (1959). New diet for laboratory animals. Brit. med.J., 1, 511-512.
Thomas, P. K. (1955). Growth changes in the myelin sheath of peripheral nerve fibres in fishes. Proc. roy. Soc. B., 143, 380-391. (1960). Motor nerve conduction in the carpal tunnel syndrome. Neurology (Minneap.), 10, 1045-1050.

and Fullerton, P. M. (1963). Nerve fibre size in the carpal tunnel syndrome. J. Neurol. Neurosurg. Psychiat., 26, 520-527. Weiss, P., and Hiscoe, H. B. (1948). Experiments on the mechanism of nerve growth. J. exp. Zool., 107, 315-395. 